\title{
Every Lusin set is undetermined in the point-open game
}

\author{
by
}

\author{
Ireneusz Recław (Gdańsk)
}

\begin{abstract}
We show that some classes of small sets are topological versions of some combinatorial properties. We also give a characterization of spaces for which White has a winning strategy in the point-open game. We show that every Lusin set is undetermined, which solves a problem of Galvin.
\end{abstract}

1. Introduction. In Set Theory many infinite combinatorial proofs are "Borel". So we can very often get interesting topological theorems using the same proofs as for combinatorics.

For example, in this paper we consider some classical notions of smallness such as the Hurewicz property, Menger property, $\mathrm{C}^{\prime \prime}$-sets and others (see [M1] and [FM]). We observe that these classes of sets can be expressed by some combinatorial properties used to define some cardinal coefficients, for example $\mathbf{b}, \mathbf{d}, \mathbf{p}, \operatorname{cov}(\mathbf{M})$. We also investigate some "measurable" versions of additivity of measure and we get some implications using the proofs of Bartoszyński (see [B1]). We apply those methods to investigate the determinacy of point-open games. In particular, we show that every Lusin set is undetermined.

We use the following notation:

- $[\omega]^{\omega}=\{A \subseteq \omega: A$ infinite $\}$,

- $\exists_{n}^{\infty}$ — there are infinitely many $n$,

- $\forall_{n}^{\infty}$ — for all but finitely many $n$,

- $s \frown t$ - the concatenation of finite sequences $s$ and $t$,

- c - the cardinal number continuum,

- $|X|$ - cardinality of $X$,

- $\mu$-Lebesgue measure,

1991 Mathematics Subject Classification: Primary 04A15, 03E05; Secondary 28A05.

Key words and phrases: point-open games, Lusin set, additivity of measure, $\gamma$-set.

Some of the results of this paper were obtained when the author was visiting Auburn University supported by The Kościuszko Foundation. 
- $G_{x}=\{y:(x, y) \in G\}$,

- $G^{y}=\{x:(x, y) \in G\}$,

- for $s \in \kappa^{<\omega}$ let $[s]=\left\{x \in \kappa^{\omega}: s \subset x\right\}$,

- $A \subset^{*} B$ if $|A \backslash B|$ is finite,

- $f<^{*} g$ if $\{n: g(n)<f(n)\}$ is finite.

We say that $X \subset \omega^{\omega}$ is bounded if there is a function $g \in \omega^{\omega}$ such that $x<^{*} g$ for each $x \in X$. We then write $X<^{*} g$.

We say that $X \subset \omega^{\omega}$ is dominating if for each $g \in \omega^{\omega}$ there is an $x \in X$ with $g<^{*} x$.

We say that a subset $L \subseteq \mathbb{R}$ is a Lusin (Sierpiński) set if $L$ is uncountable and its intersection with every set of first category (of measure zero) is countable.

We say that $X$ is concentrated on $D \subset X$ if $|X \backslash U| \leq \omega$ for every open set $U \supseteq D$.

For a property $\mathrm{H}$ let $\operatorname{non}(\mathrm{H})=\min \{|F|: F$ does not satisfy $\mathrm{H}$ and $F \subseteq \mathbb{R}\}$.

2. Games. Let $X$ be a topological space. We recall two infinite games.

The point-open game $G(X)$ : In the $n$th move Black plays a point $x_{n} \in X$ and White plays an open set $U_{n}$ containing $x_{n}$. Black wins if $\bigcup_{n} U_{n}=X$. Otherwise White wins.

$G^{*}(X)$ : In the $n$th move White plays an open cover $J_{n}$ of $X$ and Black plays an element $U_{n}$ of $J_{n}$. Black wins if $\bigcup_{n} U_{n}=X$, otherwise White wins.

TheOrem (Galvin $[\mathrm{G}]$ ). (a) $G(X)$ and $G^{*}(X)$ are equivalent.

(b) $(\mathrm{CH})$ There is a Lusin set which is undetermined.

Galvin $[\mathrm{G}]$ asked if every Lusin set is undetermined in the point-open game.

Let $\kappa=\omega(X)$ be the weight of the space $X$ with the discrete topology. Then $\kappa^{\omega}$ is a complete metric space.

THEOREM 1. White has a winning strategy in $G(X)$ iff there is a closed set $D \subseteq X \times \kappa^{\omega}$ such that $D_{x}$ is nowhere dense for every $x$ in $X$, and $\bigcup_{x \in X} D_{x}=\kappa^{\omega}$.

Proof. $\Leftarrow$ We define a winning strategy for White. At each step White chooses two open sets $U_{n}$ and $V_{n}$ such that $U_{n} \subseteq X, U_{n}$ contains $x_{n}, \bar{V}_{n} \subseteq$ $V_{n-1} \subseteq \kappa^{\omega}, \operatorname{diam}\left(V_{n}\right)<1 / n$ and $\left(U_{n} \times V_{n}\right) \cap D=\emptyset$. Then White plays $U_{n}$.

Assume that $\bigcup_{n} U_{n}=X$. Let $y \in \bigcap_{n} V_{n}$. Then $\left(U_{n} \times\{y\}\right) \cap D=\emptyset$ for each $n$. So $(X \times\{y\}) \cap D=\emptyset$, a contradiction.

$\Rightarrow$ By the Galvin Theorem, White has a winning strategy in the game $G^{*}(X)$. Let $O$ be a basis of size $\kappa$. We can assume that White chooses a 
function from $\mathcal{A}=\left\{J: \kappa \rightarrow O: \bigcup_{\alpha<\kappa} J(\alpha)=X\right\}$. So we can assume that Black chooses an $\alpha \in \kappa$.

Let $S: \kappa^{<\omega} \rightarrow \mathcal{A}$ be a strategy for White. We define

$$
W=\bigcup_{s \in \kappa<\omega} \bigcup_{\alpha \in \kappa} S(s)(\alpha) \times[s \frown\langle\alpha\rangle] .
$$

Since $W$ is open, $D=\left(X \times \kappa^{\omega}\right) \backslash W$ is closed. Let $x \in X$ and $s \in \kappa^{<\omega}$. Then there is an $\alpha \in \kappa$ such that $x \in S(s)(\alpha)$ because $S(s)$ is a cover of $X$. Then $[s \frown\langle\alpha\rangle] \cap D_{x}=\emptyset$. So $D_{x}$ is nowhere dense. If there were $y=$ $\left(\alpha_{0}, \alpha_{1}, \ldots, \alpha_{n}, \ldots\right) \in \kappa^{\omega}$ such that $y \notin \bigcup_{x \in X} D_{x}$ then Black would win playing $S\left(\alpha_{0}, \alpha_{1}, \ldots, \alpha_{n-1}\right)\left(\alpha_{n}\right)$ in the $n$th move. Observe that if $x \in X$ then $(x, y) \in W$ and so there are $s \in \kappa^{<\omega}$ and $\alpha \in \kappa$ such that $(x, y) \in S(s)(\alpha) \times$ $[s \frown\langle\alpha\rangle]$. Since $y \in[s \frown\langle\alpha\rangle]$, there is an $n \in \omega$ such that $\left(\alpha_{0}, \alpha_{1}, \ldots, \alpha_{n}\right)=$ $s \frown\langle\alpha\rangle$ so $x \in S\left(\alpha_{0}, \alpha_{1}, \ldots, \alpha_{n-1}\right)\left(\alpha_{n}\right)$.

Remarks. It is consistent that for any uncountable metric space White has a winning strategy so every metric space is determined. Telgársky constructed an uncountable, undetermined, Hausdorff, Lindelöf space. For Lindelöf spaces, the $\kappa$ in Theorem 1 can also be equal to $\omega$.

Lemma 1. Let $L \subseteq \omega^{\omega}$ be a Borel image of a Lusin set. Then there is a function $f \in \omega^{\omega}$ such that $\forall_{x \in L} \exists_{n}^{\infty}\left(x(n)=f(n) \wedge \forall_{i<n} f(i)<n\right)$.

Proof. Every Borel image of a Lusin set is concentrated on a countable subset so it is a $\mathrm{C}^{\prime \prime}$-set (for definition see the next section). So there is a function $g \in \omega^{\omega}$ such that $\forall_{x \in L} \exists_{n}^{\infty} x(n)=g(n)$. Since $g$ itself need not satisfy the assertion of the theorem, we improve it by putting in some places 0 instead of $g(n)$.

Let $\left\{y_{l}: l \in \omega\right\} \subseteq L$ be such that $L$ is concentrated on it. Inductively we can construct an increasing sequence $n_{k}$ such that $n_{k+1}>\max \{g(n): n \leq$ $\left.n_{k}\right\}$ and $\forall_{l \in \omega} \exists_{k}^{\infty} y_{l}\left(n_{k}\right)=g\left(n_{k}\right)$.

Let $K=\left\{x \in L: \exists_{k}^{\infty} x\left(n_{k}\right)=g\left(n_{k}\right)\right\}$. Observe that $K$ is a relative $G_{\delta}$ in $L$ containing $\left\{y_{l}: l \in \omega\right\}$ so $|L \backslash K| \leq \omega$.

We define a function $F: K \rightarrow \omega^{\omega}$ by $F(x)(n)=n$th element of the set $\left\{k: g\left(n_{k}\right)=x\left(n_{k}\right)\right\}$. Obviously $\left\{k: g\left(n_{k}\right)=x\left(n_{k}\right)\right\}$ is infinite for $x \in K$. Since $F$ is Borel, $F[K]$ is a Borel image of a Lusin set so there is an increasing function $a \in \omega^{\omega}$ such that $\forall_{x \in K} \exists_{i}^{\infty} F(x)(i)<a(i)$. Let $L \backslash K=\left\{z_{l}: l \in \omega\right\}$. We choose $m_{k}$ such that $m_{k}>n_{3 a(k)+3}$ and $\forall_{l \in \omega} \exists_{k}^{\infty} g\left(m_{k}\right)=z_{l}\left(m_{k}\right)$.

Now we define a function $f$ by

$$
f(n)= \begin{cases}0 & \text { if } n \notin\left\{m_{k}: k \in \omega\right\} \cup\left\{n_{k}: k \in \omega\right\}, \\ g(n) & \text { if } \exists_{k} n=m_{k}, \\ g(n) & \text { if } \exists_{k}\left(n=n_{k} \wedge \exists_{l} n_{k}<m_{l}<n_{k+1}\right), \\ 0 & \text { if } \exists_{k}\left(n=n_{k} \wedge \exists_{l} n_{k}<m_{l}<n_{k+1}\right) .\end{cases}
$$


Let $x \in L$. First assume that $x \in L \backslash K$. Then $\exists_{k}^{\infty} g\left(m_{k}\right)=x\left(m_{k}\right)$. Let $n<m_{k}$ and let $l \in \omega$ be such that $n_{l}<m_{k}<n_{l+1}$. Then if $n \leq n_{l-1}$ then $f(n) \leq g(n)<n_{l}<m_{k}$. If $n_{l-1}<n<m_{k}$ then $f(n)=0<m_{k}$.

Now assume that $x \in K$. Then $\exists_{k}^{\infty} F(x)(k) \leq a(k)$. Since $m_{[k / 3]}>a(k)$ there is an $l \leq k$ such that $\neg\left(\left(\exists_{i} n_{F(x)(l)}<m_{i}<n_{F(x)(l)+1}\right) \vee\left(n_{F(x)(l)-1}<\right.\right.$ $\left.\left.m_{i}<n_{F(x)(l)}\right)\right)$. Then $f\left(n_{F(x)(l)}\right)=g\left(n_{F(x)(l)}\right)$ and $\forall_{i \leq n_{F(x)(l)-1}} f(i) \leq$ $g(i)<n_{F(x)(l)}$ and $\forall_{i}\left(\left(n_{F(x)(l)-1}<i<n_{F(x)(l)}\right) \Rightarrow f(i)=0\right)$.

Theorem 2. Let $X \subseteq 2^{\omega}$ be a Lusin set and $D \subseteq X \times 2^{\omega}$ be a closed set such that $D_{x}$ is nowhere dense for each $x \in X$. Then $\bigcup_{x \in X} D_{x} \neq 2^{\omega}$.

Pr o of. We closely follow the line of reasoning from [M4].

Let $2^{<\omega}=\left\{s_{0}, s_{1}, \ldots\right\}$. We define $H: X \rightarrow \omega^{\omega}$ by

$$
\begin{aligned}
& H(x)(n) \\
& \quad=\min \left\{k: \forall_{i_{0}, i_{1}, \ldots, i_{n-1}<n}\left[s_{i_{0}} \frown s_{i_{1}} \frown \ldots \frown s_{i_{n-1}} \frown s_{k}\right] \cap D_{x}=\emptyset\right\} .
\end{aligned}
$$

It is easy to see that $H$ is a Borel function. Then there is a function $f \in \omega^{\omega}$ such that $\forall_{z \in H[X]} \exists_{n}^{\infty}\left(z(n)=f(n) \wedge \forall_{i<n} f(i)<n\right)$. Let $y=$ $s_{f(0)} \frown s_{f(1)} \frown \ldots \frown s_{f(n)} \frown \ldots$ We will show that $\forall_{x \in X} y \notin D_{x}$. For $z \in H[X]$, $\exists_{n}\left(z(n)=f(n) \wedge \forall_{i<n} f(i)<n\right)$. Observe that $y \in\left[s_{f(0)} \frown s_{f(1)} \frown \ldots \frown s_{f(n)}\right]$ $=\left[s_{f(0)} \frown s_{f(1)} \frown \ldots \frown s_{H(x)(n)}\right]$. So since $f(0), f(1), \ldots, f(n-1)<n$ we have $\left[s_{f(0)} \frown s_{f(1)} \frown \ldots \frown s_{f(n)}\right] \cap D_{x}=\emptyset$.

Corollary 1. Let $X \subseteq 2^{\omega}$ be a Lusin set and $D \subseteq X \times \omega^{\omega}$ be a closed set such that $D_{x}$ is nowhere dense for each $x \in X$. Then $\bigcup_{x \in X} D_{x} \neq \omega^{\omega}$.

Proof. $\omega^{\omega}$ is homeomorphic to a subset $Z \subseteq 2^{\omega}$ such that $2^{\omega} \backslash Z$ is countable. If $\bigcup_{x \in X} D_{x}=\omega^{\omega}$ then by a natural homeomorphism we can construct a set $C \subseteq X \times 2^{\omega}$ such that $C_{x} \supseteq Z$. Then adding to $X$ countably many isolated points we can obtain the missing points from $2^{\omega}$ to get a contradiction with Theorem 2 .

So we get a positive answer to the question of Galvin.

COROLlary 2. Every Lusin set is undetermined in the point-open game.

Proof. By Theorem 1, White does not have a winning strategy for a Lusin set. For subsets of the reals Black has a winning strategy only for countable sets so every Lusin set is undetermined.

Corollary 3. Let $X \subseteq 2^{\omega}$ be a Lusin set and $D \subseteq X \times \mathbb{R}$ be a first category set in $X \times \mathbb{R}$ such that $D_{x}$ is first category for each $x \in X$. Then $\bigcup_{x \in X} D_{x} \neq \mathbb{R}$.

Proof. Observe that for every closed set $D \subseteq X \times \mathbb{R}$ with every section nowhere dense, $\bigcup_{x \in X} D_{x}$ is not residual. Otherwise we could obtain $\mathbb{R}$ adding countably many isolated points to $X$. 
First assume that $D=\bigcup_{n} D_{n}$ is such that $D_{n}$ is closed with every section nowhere dense. Define $C=\left\{(n, x, y):(x, y) \in D_{n}\right.$ and $\left.n \in \omega\right\}$. Observe that $C$ is closed, $\{n\} \times X$ is a Lusin set and $C_{(n, x)}=\left(D_{n}\right)_{x}$.

Now let $D \subseteq X \times \mathbb{R}$ be a first category set in $X \times \mathbb{R}$ such that each $D_{x}$ is first category. Then $D$ is contained in a $F_{\sigma}$-set $F$ of first category. The set $A=\left\{x: F_{x}\right.$ is second category $\}$ is countable. Since $X \backslash A$ is also a Lusin set, $\bigcup_{x \in X \backslash A} F_{x}$ is not residual. Thus $\bigcup_{x \in X} D_{x} \subseteq \bigcup_{x \in A} D_{x} \cup \bigcup_{x \in X \backslash A} F_{x} \neq \mathbb{R}$.

3. Small sets and cardinal coefficients. In this section we will compare some notions of smallness in the sense of topology and combinatorics. Definitions 1-4 can be found in [FM], and Definition 6 in [GM]. The definitions of the coefficients $\mathbf{p}, \mathbf{d}, \mathbf{b}$ are in $[\mathrm{D}] \cdot \operatorname{cov}(\mathbf{M})$ and $\operatorname{add}(\mathbf{N})$ were investigated for example in [B1] and [M5].

Definition 1. A topological space $X$ has the Hurewicz property if for every family $\left\{J_{n}: n \in \omega\right\}$ of open covers of $X$ there is a family $\left\{J_{n}^{\prime}: n \in \omega\right\}$ such that $J_{n}^{\prime}$ is a finite subset of $J_{n}$ and $X \subseteq \bigcup_{k} \bigcap_{n>k} \cup J_{n}^{\prime}$.

Proposition 1. Let $X$ be a 0-dimensional, separable metric space. Then $X$ has the Hurewicz property iff every continuous image of $X$ into $\omega^{\omega}$ is bounded.

Pr o of. $\Rightarrow$ Every continuous image of a Hurewicz set is a Hurewicz set. Let $X$ be a subset of $\omega^{\omega}$. Let $J_{n}=\left\{\left\{f \in \omega^{\omega}: f(n)=k\right\}: k \in \omega\right\}$. By the Hurewicz property this set must be bounded.

$\Leftarrow$ Let $J_{n}$ be a family of open covers of $X$. By 0 -dimensionality we can assume that all elements of $J_{n}$ are clopen and disjoint. Define $h: X \rightarrow \omega^{\omega}$ by $h(x)(n)=k$ if $x$ belongs to the $k$ th element of $J_{n}$. Then $h$ is continuous and $h[X]$ is bounded so there is a $\phi \in \omega^{\omega}$ such that $h[X] \leq{ }^{*} \phi$. Then $J_{n}^{\prime}$ is simply the first $\phi(n)$ elements of $J_{n}$.

As in Proposition 1, we will consider other pairs of classes of small sets and coefficients.

We say that a family $J$ of subsets of $X$ is an $\omega$-cover if for each finite set $A \subset X$ there is a $U \in J$ such that $A \subset U$.

Definition 2. $X$ is a $\gamma$-set if for every open $\omega$-cover $J$ of $X$ there exists a sequence $\left(D_{n}: n \in \omega\right)$ of elements of $J$ such that $X \subseteq \bigcup_{k} \bigcap_{n>k} D_{n}$.

We say that $F \subseteq \omega^{\omega}$ has property $\mathrm{P}$ if $\left|\cap F_{0}\right|=\omega$ for every finite subset $F_{0}$ of $F$. Then there exists $A \in[\omega]^{\omega}$ such that $A \subseteq{ }^{*} B$ for every $B \in F$. We define

$$
\mathbf{p}=\min \left\{|F|: F \in[\omega]^{\omega} \text { and } \neg(F \text { has property } \mathrm{P})\right\} .
$$

Proposition 2. Let $X$ be a 0 -dimensional, separable metric space. Then $X$ is a $\gamma$-set iff $f[X]$ has property $\mathrm{P}$ for every continuous function $f: X \rightarrow$ $[\omega]^{\omega}$. 
Proof. $\Rightarrow$ Every continuous image of a $\gamma$-set is a $\gamma$-set. Let $X$ be a subset of $[\omega]^{\omega}$. Let $O_{n}=\left\{A \in[\omega]^{\omega}: n \in A\right\}$. Assume that $\left|\cap F_{0}\right|=\omega$ for every finite subset $F_{0}$ of $X$. Then the family $J=\left\{O_{n}: n \in \omega\right\}$ is an open $\omega$-cover of $X$. By the $\gamma$-property there is a sequence $n_{k}$ such that $X \subseteq \bigcup_{m} \bigcap_{k>m} O_{n_{k}}$. The case when the sequence $n_{k}$ has only finitely many values is left to the reader. Assume that $n_{k}$ is increasing. Then for every $B \in X$ almost every $n_{k}$ belongs to $B$. Thus $\left\{n_{k}: k \in \omega\right\} \subseteq^{*} B$.

$\Leftarrow$ Let $J=\left\{D_{n}: n \in \omega\right\}$ be an open $\omega$-cover of $X$. By 0-dimensionality we can assume that all elements of $J$ are clopen and every subset of $X$ is contained in infinitely many elements of $J$. Define $h: X \rightarrow[\omega]^{\omega}$ by $h(x)=A$ iff (for every $n, n \in A$ iff $x \in D_{n}$ ). Then $h$ is continuous and $\left|\bigcap F_{0}\right|=\omega$ for every finite subset $F_{0}$ of $h[X]$. So there exists $A \in[\omega]^{\omega}$ such that $A \subseteq \subseteq^{*} B$ for every $B \in h[X]$. We can see that $h^{-1}\left[O_{n}\right]=D_{n}$. So $X \subseteq \bigcup_{m} \bigcap_{k>m} D_{n_{k}}$ where $\left\{n_{k}: k \in \omega\right\}=A$.

Definition 3. $X$ has the Menger property if for every sequence $\left(J_{n}\right.$ : $n \in \omega)$ of open covers there is a sequence $\left(J_{n}^{\prime}: n \in \omega\right)$ such that $J_{n}^{\prime} \subseteq J_{n}$, $J_{n}^{\prime}$ is finite, and $X \subseteq \bigcup_{n} \cup J_{n}^{\prime}$.

We set

$$
\mathbf{d}=\min \left\{|F|: F \subseteq \omega^{\omega} \text { and } \neg(F \text { is not dominating })\right\} .
$$

Proposition 3. Let $X$ be a 0-dimensional, separable metric space. Then $X$ has the Menger property iff for every continuous function $f: X \rightarrow$ $\omega^{\omega}, f[X]$ is not dominating.

Proof. The proof is similar to the proof of Proposition 1.

Definition 4. $X$ has the $\mathrm{C}^{\prime \prime}$ property if for every sequence $\left(J_{n}: n \in \omega\right)$ of open covers there is a sequence $\left(D_{n}: n \in \omega\right)$ such that $D_{n} \in J_{n}$ and $X \subseteq \bigcup_{n} D_{n}$.

We set

$$
\operatorname{cov}(\mathbf{M})=\min \{|F|: F \subseteq \mathbf{M} \text { and } \bigcup F=\mathbb{R}\}
$$

where $\mathbf{M}$ is the $\sigma$-ideal of first category sets.

We say that $F \subseteq \omega^{\omega}$ has property $\mathrm{CM}$ if there exists $g \in \omega^{\omega}$ such that for every $f \in F$ there exist infinitely many $n$ such that $f(n)=g(n)$.

Bartoszyński [B2] showed that $\operatorname{cov}(\mathbf{M})=\left\{|F|: F \subseteq \omega^{\omega}\right.$ and $\neg(F$ has property $\mathrm{CM})\}$.

Proposition 4. Let $X$ be a 0-dimensional, separable metric space. Then $X$ is a $\mathrm{C}^{\prime \prime}$-set iff $f[X]$ has property $\mathrm{CM}$ for every continuous function $f$ : $X \rightarrow \omega^{\omega}$.

Proof. The proof is similar to the proof of Proposition 1. 
We also set

$$
\operatorname{add}(\mathbf{N})=\{|F|: F \subseteq \mathbf{N} \text { and } \bigcup F \notin \mathbf{N}\}
$$

where $\mathbf{N}$ is the $\sigma$-ideal of Lebesgue measure zero sets.

Let $k_{n}$ be an arbitrary increasing sequence of natural numbers. We say that $F \subseteq \omega^{\omega}$ has property AN if there exists $g \in\left([\omega]^{<\omega}\right)^{\omega}$ such that $|g(n)| \leq$ $k_{n}$ for every $n$, and $f(n) \in g(n)$ for every $f \in F$ and for almost every $n$.

Bartoszyński [B1] showed that $\operatorname{add}(\mathbf{N})=\min \left\{|F|: F \subseteq \omega^{\omega}\right.$ and $\neg(F$ has property $\mathrm{AN})\}$.

Definition 5. We say that $X$ is $\operatorname{add}(\mathbf{N})$-small if there exists an increasing sequence $k_{n}$ such that for every sequence $\left(J_{n}: n \in \omega\right)$ of open covers there is a sequence $\left(J_{n}^{\prime}: n \in \omega\right)$ such that $J_{n}^{\prime} \subseteq J_{n},\left|J_{n}^{\prime}\right| \leq k_{n}$, and $X \subseteq \bigcup_{k} \bigcap_{n>k} \cup J_{n}^{\prime}$.

Proposition 5. Let $X$ be a 0-dimensional, separable metric space. Then $X$ is add(N)-small iff $f[X]$ has property $\mathrm{AN}$ for every continuous function $f: X \rightarrow \omega^{\omega}$.

Proof. The proof is similar to the proof of Proposition 1.

We say that $J$ is a cover of $[X]^{k}$ if for every finite set $A \subset X$ of size $k$ there is an element $O \in J$ with $A \subset O$.

Definition 6. We say that $X$ is a strong $\gamma$-set iff there exists $\left(k_{n}: n \in\right.$ $\omega)$ such that for any sequence $\left(J_{n}: n \in \omega\right)$ where $J_{n}$ is an open cover of $[X]^{k_{n}}$ there exists $\left(C_{n}: n \in \omega\right)$ with $C_{n} \in J_{n}$ and $X \subseteq \bigcup_{n} \bigcap_{m>n} C_{m}$.

Proposition 6. Every strong $\gamma$-set is $\operatorname{add}(\mathbf{N})$-small.

Proof. Let $\left(I_{n}: n \in \omega\right)$ be a family of open covers of a strong $\gamma$-set $X$, and $J_{n}=\left\{\bigcup I_{n}^{\prime}: I_{n}^{\prime} \subseteq I_{n}\right.$ and $\left.\left|I_{n}^{\prime}\right| \leq k_{n}\right\}$. Then $J_{n}$ is a cover of $[X]^{k_{n}}$. Since $X$ is a strong $\gamma$-set there is a sequence $\left(D_{n}: n \in \omega\right)$ such that $D_{n} \in J_{n}$ and $X \subseteq \bigcup_{n} \bigcap_{m>n} D_{m}$. Since we know that $D_{n}$ is a union of at most $k_{n}$ elements of $I_{n}$ we conclude that $X$ is $\operatorname{add}(\mathbf{N})$-small.

From the existence of a strong $\gamma$-set of size $\mathbf{c}$ under MA we get:

COROllary 4. Assuming Martin's Axiom there exists an $\operatorname{add}(\mathbf{N})$-small set of reals of size $\mathbf{c}$.

Pr o o f. See $[\mathrm{GM}]$ for an example of a strong $\gamma$-set.

Remarks. From the results above we find that: $\operatorname{non}(\gamma)=\mathbf{p}$, non $(\mathrm{Hu}-$ rewicz property $)=\mathbf{b}$, non $($ Menger property $)=\mathbf{d}$, non $\left(\mathrm{C}^{\prime \prime}\right)=\operatorname{cov}(\mathbf{M})$ and $\operatorname{non}(\operatorname{add}(\mathbf{N})$-small $)=\operatorname{add}(\mathbf{N})$. These results except the last one were obtained in $[\mathrm{FM}]$. 
The following relations between the cardinal coefficients mentioned above are known: $\operatorname{add}(\mathbf{N}) \leq \operatorname{cov}(\mathbf{M}) \leq \mathbf{d}$ and $\mathbf{p} \leq \mathbf{b} \leq \mathbf{d}$ and $\mathbf{p} \leq \operatorname{cov}(\mathbf{M})$. This is a part of Cichoń's Diagram (see $[\mathrm{F}]$ ).

Similar inclusions exist for classes of sets: (strong $\gamma \rightarrow \gamma \rightarrow$ Hurewicz property $\rightarrow$ Menger property) and ( $\gamma \rightarrow \mathrm{C}^{\prime \prime} \rightarrow$ Menger property).

We can see that non $($ strong $\gamma) \leq \min (\operatorname{add}(\mathbf{N})$, p). So if $\operatorname{add}(\mathbf{N})<\mathbf{p}$, what is consistent, then strong $\gamma \neq \gamma$. This fact was observed before by T. Weiss (private communication) in a more particular model of ZFC.

4. "Measurable" additivity and non-covering. In [B1] Bartoszyński introduced several equivalent conditions for additivity of measure. We will translate some of them to "measurable" versions. We will also show some implications between them. A first condition can be found in Definition 5 . Below we consider some other properties of a set $X$ :

(*) $\quad$ Let $V \subseteq \mathbb{R}^{2}$ be such that $V \subseteq \bigcap_{n} U_{n}$ where $U_{n}$ is open and $\mu\left(\left(U_{n}\right)_{x}\right)<$ $2^{-n}$ for all $x \in X$. Then $\mu\left(\bigcup_{x \in X} V_{x}\right)=0$.

It is easy to see that every set $X \subseteq \mathbb{R}$ with property (*) also has the following property: For every $G$ of measure zero, $X+G$ is also of measure zero. Sets with this property were investigated for example in [GM] and [FJ].

$(* *) \quad$ For every sequence of continuous functions $f_{n}: X \rightarrow \mathbb{R}$ such that the series $\sum f_{n}$ is converging there is a convergent series $\sum a_{n}$ eventually dominating $\left(f_{n}: n \in \omega\right)$ (that is, $\left.\forall_{x} \exists_{k} \forall_{n>k}\left|f_{n}(x)\right|<a_{n}\right)$.

Proposition 7. $(* *) \Rightarrow(*)$.

Proof. The proof uses similar arguments to a proof in [B1].

Let $U_{n}=\bigcup_{k} P_{n k} \times Q_{n k}$ be such that the $P_{n k} \times Q_{n k}$ are pairwise disjoint for each $n$, the $P_{n k}$ are clopen and the $Q_{n k}$ are intervals. Let us enumerate $\left\{P_{n k} \times Q_{n k}: n, k \in \omega\right\}$ as $\left\{P_{l} \times Q_{l}: l \in \omega\right\}$. Then $V \subseteq \bigcap_{k} \bigcup_{l>k} P_{l} \times Q_{l}$.

Let $f_{l}: X \rightarrow \mathbb{R}, f_{l}=\chi_{P_{l}} \cdot \mu\left(Q_{l}\right)$. Since $\sum_{l} f_{l}<\infty$ there is a convergent series $\sum a_{l}$ such that $\forall_{x} \exists_{k} \forall_{l>k}\left|f_{l}(x)\right|<a_{l}$. We define

$$
R_{l}= \begin{cases}Q_{l} & \text { if } \mu\left(Q_{l}\right)<a_{l}, \\ \emptyset & \text { otherwise. }\end{cases}
$$

Observe that $\bigcap_{k} \bigcup_{l>k} R_{l}$ is a null set. We know that $V_{x} \subseteq \bigcap_{k} \bigcup_{l>k, x \in P_{l}} Q_{l}$ for every $x$. Observe that for every $x$ and almost every $l$ if $x \in P_{l}$ then $Q_{l}=R_{l}$ so $V_{x} \subseteq \bigcap_{k} \bigcup_{l>k} R_{l}$.

We next define two properties of a set $X$ :

$(* * *) \quad \forall f_{n}: X \rightarrow \mathbb{R}$ Borel

$$
\left(\sum f_{n}<\infty\right) \Rightarrow \exists_{a_{n}}\left(\sum a_{n}<\infty \text { and } \forall_{n}^{\infty}\left|f_{n}(x)\right|<a_{n}\right) .
$$

$(* * * *) \quad \forall_{g: X \rightarrow \omega^{\omega}}$ Borel $\exists_{I_{n} \subset \omega}\left|I_{n}\right|<n^{2} \wedge \forall_{h \in g[X]} \forall_{n}^{\infty} h(n) \in I_{n}$. 
It is obvious that $(* * *) \Rightarrow(* *)$.

Proposition 8. $(* * * *) \Rightarrow(* * *)$.

Proof. The proof is a slight modification of a proof from [B1].

Let $g: X \rightarrow \omega^{\omega}, g(x)(k)=\min \left\{n: \sum_{l>n}\left|f_{l}(x)\right|<2^{-k}\right\}$. Then $g$ is a Borel function. So there is a sequence $b_{k}$ such that $\forall_{k}^{\infty} \forall_{n \geq b_{k}} \sum_{l>n}\left|f_{l}(x)\right|<$ $2^{-k}$. We can assume that $f_{n}[X] \subseteq \mathbb{Q}$ for each $n$. Let $g_{1}: X \rightarrow\left(\mathbb{Q}^{<\omega}\right)^{\omega}$ where we take $\mathbb{Q}^{<\omega}$ with discrete topology such that $g_{1}(x)(k)=\left(f_{b_{k}}(x), f_{b_{k}+1}(x)\right.$, $\left.\ldots, f_{b_{k+1}-1}(x)\right)$. Since $g_{1}$ is a Borel function, there are $I_{k} \subseteq \mathbb{Q}^{<\omega}$ such that $\left|I_{k}\right|<k^{2}$ and $\forall_{h \in g_{1}} \forall_{k}^{\infty} h(k) \in I_{k}$. Define $a_{n}=\sup \left\{f_{n}(x):\left(f_{b_{k}}(x), f_{b_{k}+1}(x)\right.\right.$, $\left.\ldots, f_{b_{k+1}-1}(x)\right) \in I_{k}$ and $\sum_{l=b_{k}}^{b_{k+1}-1}\left|f_{l}(x)\right|<2^{-k}$ and $x \in X$ and $b_{k} \leq n \leq$ $\left.b_{k+1}-1\right\}$. It is easy to see that $\sum_{l=b_{k}}^{b_{k+1}-1} a_{l} \leq k^{2} 2^{-k}$ so $a_{n}$ is convergent and eventually dominates each $f_{n}$.

FACT. $\operatorname{non}((*))=\operatorname{non}((* *))=\operatorname{non}((* * *))=\operatorname{non}((* * * *))=\operatorname{add}(\mathbf{N})$.

Proof. It is enough to show that $\operatorname{add}(\mathbf{N}) \geq \operatorname{non}((*))$.

Let $\left\{G_{x}: x \in X\right\}$ be a family of $G_{\delta}$-sets of measure zero such that $\mu^{*}\left(\bigcup_{x \in X} G_{x}\right)>0$. Let $\left\{U_{x}^{n}: n \in \omega\right\}$ be a family of open sets such that $G_{x}=\bigcap_{n} U_{x}^{n}$ and $\mu\left(U_{x}^{n}\right)<1 / n$. Now we can find a separable, 0-dimensional metric topology such that $\bigcup_{x \in X}\{x\} \times U_{x}^{n}$ is an open set in $X \times \mathbb{R}$ for each $n$ (see $[\mathrm{BBM}])$. So we see that $X$ does not satisfy $(*)$.

Proposition 9. Suppose that every Borel image of a set $X$ into $\omega^{\omega}$ is bounded. Let $B \subseteq X \times \mathbb{R}$ be a Borel set. Then there is a sequence of open sets $U_{n} \subseteq X \times \mathbb{R}$ such that $B \subseteq \bigcap_{n} U_{n}$ and $\forall_{x \in X} \forall_{n}^{\infty} \mu\left(\left(U_{n} \backslash B\right)_{x}\right)<2^{-n}$.

Proof. First we show that the family $\mathcal{A}$ of all Borel sets $A \subseteq X \times \mathbb{R}$ for which there is a sequence of open subsets $U_{n}$ of $X \times \mathbb{R}$ such that $A \subseteq \bigcap_{n} U_{n}$ and $\forall_{x \in X} \forall_{n}^{\infty} \mu\left(\left(U_{n} \backslash A\right)_{x}\right)<2^{-n}$ is a monotonic family. Let $A=\bigcap_{k} A^{k}$ and let $U_{n}^{k}$ witness that $A^{k} \in \mathcal{A}$.

We define $f: X \rightarrow \omega^{\omega}$ by $f(x)(k)=\min \left\{n: \forall_{l \geq n} \mu\left(\left(U_{l}^{k} \backslash A_{k}\right)_{x}\right)<2^{-k}\right\}$. Since $f$ is Borel, there is an $h \in \omega^{\omega}$ with $f[X]<^{*} h$. Let $g: X \rightarrow \omega^{\omega}$ be defined by $g(x)(i)=\min \left\{n: \forall_{k \geq n} \mu\left(\left(A^{k} \backslash A\right)_{x}\right)<2^{-i}\right\}$. Then $g$ is Borel so there is an $\phi \in \omega^{\omega}$ with $g[X]<^{*} \phi$. Then $U_{h(\phi(k))}^{\phi(k)}$ has the required properties.

Now let $A=\bigcup_{k} A^{k}$ with $A^{k} \subseteq A^{k+1}$ and let $U_{n}^{k}$ witness that $A^{k} \in \mathcal{A}$. We define $f: X \rightarrow \omega^{\omega}$ by $f(x)(k)=\min \left\{n: \forall_{l \geq n} \mu\left(\left(U_{l}^{k} \backslash A_{k}\right)_{x}\right)<2^{-k}\right\}$. Since $f$ is Borel, there is an $h \in \omega^{\omega}$ with $f[X]<^{*} h$. Then the family $V_{n}=\bigcup_{l \geq n} U_{h(l)}^{l}$ has the required properties.

It is easy to show that the algebra generated by rectangles (open interval cross open interval) is contained in $\mathcal{A}$ so all Borel sets belong to $\mathcal{A}$.

Corollary 5. If $X$ satisfies (****) then $\mu\left(\bigcup_{x \in X} B_{x}\right)=0$ for every Borel set $B \subseteq X \times \mathbb{R}$ with $\mu\left(B_{x}\right)=0$ for each $x \in X$. 
Pr o o f. Obviously property $(* * * *)$ is hereditary and (****) for $X$ implies that every Borel image of $X$ into $\omega^{\omega}$ is bounded. So there is a family of open sets $U_{n}$ such that $\forall_{x \in X} \forall_{n}^{\infty} \mu\left(U_{n}\right)<2^{-n}$ and $B \subseteq \bigcap_{n} U_{n}$. Let $X_{k}=\{x \in$ $\left.X: \forall_{n \geq k} \mu\left(\left(U_{n}\right)_{x}\right)<2^{-n}\right\}$. Then $\bigcup_{k} X_{k}=X$. By Propositions 7 and 8 , $\mu\left(\bigcup_{k} \bigcup_{x \in X_{k}} B_{x}\right)=0$.

EXAMPLES. Every set of size less than add(N) satisfies (****).

Another example comes from a result of Todorčević. Since it has not been published we will sketch the proof.

Theorem 3 (Todorčević). Assuming $C H$ there is a set of size $\mathbf{c}$ whose every Borel image is a strong $\gamma$-set.

Lemma 2. Let $\left\{B_{n}: n \in \omega\right\}$ be a family of disjoint perfect sets, $A$ a countable set and $\left(J_{n}: n \in \omega\right)$ a sequence of countable Borel families such that $J_{n}$ is a cover of $\left[\bigcup_{l} D_{l} \cup A\right]^{k_{n}}$. Then there is a family $\left\{B_{n}^{\prime}: n \in \omega\right\}$ of perfect sets with $B_{n}^{\prime} \subseteq B_{n}$ and a sequence $\left\{D_{n}: n \in \omega\right\}$ with $D_{n} \in J_{n}$ such that $\bigcup_{n} B_{n}^{\prime} \cup A \subseteq \bigcup_{m} \bigcap_{n>m} D_{n}$.

Pr o of. The proof uses similar argument to the proof of a lemma in [GM].

Proof of Theorem 3. We construct an Aronszajn tree built from perfect sets of $\mathbb{R}$ ordered by reverse inclusion. First we order all pairs $\left(f_{\alpha},\left(I_{n}^{\alpha}: n \in \omega\right)\right)$ where $f_{\alpha}: \mathbb{R} \rightarrow \mathbb{R}$ is a Borel function and each $I_{n}$ is a family of open subsets of $\mathbb{R}$. Let $J_{n}^{\alpha}=\left\{f_{\alpha}^{-1}(O): O \in I_{n}^{\alpha}\right\}$. On each level $\alpha$ we extend the tree by constructing a countable family of perfect sets and a countable subset $X_{\alpha}$ of their union either using Lemma 2 for $\left(J_{n}^{\alpha}: n \in \omega\right)$ or choosing $X_{\alpha}$ such that $J_{n}^{\alpha}$ is not a cover of $\left[X_{\alpha}\right]^{k_{n}}$ for some $n$. Then $X=\bigcup_{\alpha<\omega_{1}} X_{\alpha}$ has the required properties. For details see [GM].

COROllary 6. Assuming $\mathrm{CH}$ there is a set of reals of size $\mathbf{c}$ with property $(* * * *)$.

Corollary 7. Assuming $C H$ there is a set of reals of size $\mathbf{c}$ such that $\bigcup_{x \in X} \mu\left(B_{x}\right)=0$ for every Borel set $B \subseteq X \times \mathbb{R}$ with $\mu\left(B_{x}\right)=0$ for each $x \in X$.

D. H. Fremlin and J. Jasiński [FJ] showed that if Martin's Axiom holds and there exists $\kappa<\mathbf{c}$ such that $P(\kappa)$ contains a proper uniform $\omega_{1^{-}}$ saturated $\kappa$-additive ideal then there exists a set $X$ of reals of cardinality $\mathbf{c}$ containing a subset $D$ of cardinality less than $\mathbf{c}$, Borel-dense in $X$. It is easy to show that this set also satisfies $(* * * *)$.

It is obvious that $(* * * *)$ implies that $X$ is $\operatorname{add}(\mathbf{N})$-small.

Definition 7. A set $X \subseteq \mathbb{R}$ is strong first category (= strongly meagre) (see [M1]) if for every set $G \subseteq \mathbb{R}$ of measure zero there is a $t \in \mathbb{R}$ such that $X \cap(G+t)=\emptyset$ (or equivalently $X+G \neq \mathbb{R}$ ). 
We now consider another property of a set $X$ :

(+) For every Borel set $H \subseteq \mathbb{R}^{2}$ such that $\mu\left(H_{x}\right)=0$ for every $x \in X$, we have $\bigcup_{x \in X} H_{x} \neq \mathbb{R}$.

Observe that $(+) \Rightarrow$ strong first category.

There is a question of Galvin (see [M1]) whether every Sierpiński set is strong first category. We can also raise the question whether every Sierpiński set has $(+)$.

It is known that under $\mathrm{CH}$ there is a Sierpiński set which is strong first category. We show a stronger example:

Proposition 10. Under the Continuum Hypothesis there is a Sierpinski set with $(+)$.

Proof. Let $H_{\alpha}, \alpha<\mathbf{c}$, be all Borel sets on the plane such that $\mu\left(\left(H_{\alpha}\right)_{x}\right)$ $=0$ for every $x \in \mathbb{R}$, and let $G_{\alpha}, \alpha<\mathbf{c}$, be all Borel subsets of the reals of measure zero. We define inductively sequences $\left\{x_{\alpha}, y_{\alpha}: \alpha<\mathbf{c}\right\}$.

Let $y_{\alpha} \notin \bigcup_{\beta<\alpha}\left(H_{\alpha}\right)_{x_{\beta}}$ be such that $\mu\left(\mathbb{R} \backslash\left(H_{\alpha}\right)^{y_{\alpha}}\right)=0$. Let $x_{\alpha} \in \mathbb{R} \backslash$ $\bigcup_{\beta<\alpha}\left(G_{\beta} \cup\left(H_{\beta}\right)^{y_{\beta}}\right)$. Observe that $y_{\alpha} \notin \bigcup_{\beta<\mathbf{c}}\left(H_{\alpha}\right)_{x_{\beta}}$ for every $\alpha$.

Re mark. It is easy to see that no set with $(+)$ can be mapped onto the reals by a Borel function. In $[R]$ under MA there is constructed a strong first category set which can be mapped onto the reals by a continuous function. So under MA strong first category does not imply $(+)$.

Let $\mathbf{N}_{2}$ be the ideal of null sets on the plane and $\mathbf{M}_{2}$ the ideal of meagre sets on the plane.

Problem. (CH) Do there exist functions $f, g: \mathbb{R} \rightarrow \mathbb{R}$ such that $(f, g)$ : $\mathbb{R}^{2} \rightarrow \mathbb{R}^{2}$ satisfies $(f, g)\left[\mathbf{N}_{2}\right]=\mathbf{M}_{2}$ ?

A simpler question is also interesting: $(\mathrm{CH})$ Let $G \subseteq \mathbb{R}^{2}$ be a set of measure zero. Do there exist functions $f, g: \mathbb{R} \rightarrow \mathbb{R}$ such that $(f, g)[G]$ is first category and $f[\mathbf{N}]=\mathbf{M}$ and $g[\mathbf{N}]=\mathbf{M}$ ?

Observe that the positive answer to one of the above questions and Corollary 3 show that every Sierpiński set has $(+)$ so give the solution to the problem of Galvin about Sierpiński sets.

Re m ark. In $[\mathrm{PR}]$ the authors show that for every $X \subseteq \mathbb{R}$ if $\mu\left(\bigcup_{x \in X} G_{x}\right)$ $=0$ for every Borel set $G \subseteq \mathbb{R}^{2}$ with $\mu\left(G_{x}\right)=0$ for each $x \in X$, then $\bigcup_{x \in X} F_{x}$ is meagre for every Borel set $F \subseteq \mathbb{R}^{2}$ such that each $F_{x}$ is meagre.

Remark. Recently J. Pawlikowski has solved the problem of Galvin by showing that every Sierpiński set is strongly meagre. He also showed that every Sierpiński set has $(+)$.

The author would like to express his thanks to G. Gruenhage and J. Jasiński for fruitful discussions. 


\section{References}

[B1] T. Bartoszyński, Additivity of measure implies additivity of category, Trans. Amer. Math. Soc. 281 (1984), 209-213.

[B2] - Combinatorial aspects of measure and category, Fund. Math. 127 (1987), 225-239.

[BBM] R. H. Bing, W. W. Bledsoe and R. D. Mauldin, Sets generated by rectangles, Pacific J. Math. 51 (1974), 27-36.

[BRR] L. Bukovský, I. Recław and M. Repický, Spaces not distinguishing pointwise and quasinormal convergence of real functions, Topology Appl. 41 (1991), 25-40.

[D] E. K. van Douwen, The integers and topology, in: Handbook of Set-Theoretic Topology, K. Kunen and J. Vaughan (eds.), North-Holland, Amsterdam, 1984, 111-167.

[F] D. H. Fremlin, Cichoń's diagram in: Sém. Initiation à l'Analyse, G. Choquet, M. Rogalski, J. Saint-Raymond, Université Pierre et Marie Curie, Paris, 1983/84, no. 5,13 pp.

[FJ] D. H. Fremlin and J. Jasiński, $G_{\delta}$-covers and large thin sets of reals, Proc. London Math. Soc. (3) 53 (1986), 518-538.

[FM] D. H. Fremlin and A. W. Miller, On some properties of Hurewicz, Menger, and Rothberger, Fund. Math. 129 (1988), 17-33.

[G] F. Galvin, Indeterminacy of point-open games, Bull. Acad. Polon. Sci. 26 (1978), 445-449.

[GM] F. Galvin and A. W. Miller, $\gamma$-sets and other singular sets of real numbers, Topology Appl. 17 (1984), 145-155.

[M1] A. W. Miller, Special subsets of the real line, in: Handbook of Set-Theoretic Topology, K. Kunen and J. Vaughan (eds.), North-Holland, Amsterdam, 1984, 201-233.

[M2] - Some properties of measure and category, Trans. Amer. Math. Soc. 266 (1981), 93-114; Corrections and additions, ibid. 271 (1982), 347-348.

[M3] —, Additivity of measure implies dominating reals, Proc. Amer. Math. Soc. 91 (1984), 111-117.

[M4] - , The Baire category theorem and cardinals of countable cofinality, J. Symbolic Logic 47 (1982), 275-287.

[M5] - A characterization of the least cardinal for which Baire category theorem fails, Proc. Amer. Math. Soc. 86 (1982), 498-502.

[PR] J. Pawlikowski and I. Recław, On parametrized Cichon's diagram, in preparation.

[R] I. Recław, On small sets in the sense of measure and category, Fund. Math. 133 (1989), 255-260.

INSTITUTE OF MATHEMATICS

UNIVERSITY OF GDAŃSK

WITA STWOSZA 57

80-952 GDAŃSK, POLAND

Received 29 July 1992;

in revised form 15 May 1993 